\title{
Mesure par diffraction des rayons $X$ des microdéformations dans des films minces texturés d'Au
}

\author{
N. Durand, L. Bimbault, K. F. Badawi et Ph. Goudeau \\ Laboratoire de Métallurgie Physique (URA 131), Université de Poitiers, 40 avenue du Recteur \\ Pineau, 86000 Poitiers, France
}

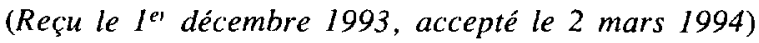

\begin{abstract}
Résumé. - Cette étude présente une analyse des microdéformations dans des films minces de $150 \mathrm{~nm}$ d'Au texturé. En appliquant la méthode de la "largeur intégrale " connue et employée dans les matériaux massifs, nous avons montré sa faisabilité et son intérêt dans le cas de couches minces. Ainsi, en relation avec la méthode de mesure des contraintes par diffraction des rayons $\mathrm{X}$, elle a permis de mettre en évidence des effets importants des conditions opératoires sur la microstructure des films.
\end{abstract}

\begin{abstract}
Microdistorsion analysis in Au $150 \mathrm{~nm}$ thin films is presented in this study. Applying the method of the "integral width", known and employed in bulk materials, we have shown its feasibility and its interest in the case of thin films. Furthermore, in relation with X-Ray diffraction stress measurement, we found important effects of the deposition conditions on the films microstructure.
\end{abstract}

\section{Introduction.}

L'influence de l'état mécanique des couches minces sur l'ensemble de leurs propriétés physiques n'est plus à démontrer [1-3, par exemple]. Ainsi, la conductivité, le magnétisme, la dureté, la résistance à l'usure, etc. sont affectés par cet état. Par ailleurs, une étude récente [4] a clairement mis en évidence une forte corrélation entre la microstructure de la phase diffractante et les contraintes résiduelles dans le cas de films minces de tungstène.

Dans cette étude, nous avons réalisé une analyse des microdéformations en déconvoluant les pics de diffraction selon la méthode de la largeur intégrale, couramment utilisée dans les matériaux massifs [5, 6]. A notre connaissance, une telle étude n'a jamais encore été appliquée au cas des couches minces. Pourtant, cette analyse donne de précieux renseignements sur la microstructure, et en association avec la méthode dite des $\sin ^{2} \Psi$ [4, 6] (qui détermine les contraintes résiduelles et le paramètre cristallin sans contraintes de la phase diffractante du film), elle permet d'espérer une meilleure compréhension des mécanismes microscopiques de la genèse et de la relaxation des contraintes.

Après avoir présenté la méthode d'analyse des microdéformations nous donnerons les premiers résultats expérimentaux obtenus en utilisant le rayonnement synchroton du L.U.R.E. à Orsay (France). 


\section{Analyse des microdéformations.}

En première approximation, on relie souvent la largeur d'un pic de diffraction (corrigé de l'élargissement instrumental) à la taille des grains diffractants. Ainsi, la formule de Scherrer [7], est souvent appliquée. En réalité, cette approche ne prend pas en compte les distorsions du réseau cristallin qui peuvent s'avérer très importantes dans le cas de grains nanométriques et entraîner une détermination erronée de la taille des domaines cohérents de diffraction. En effet, les microdéformations induisent également un élargissement du pic de diffraction. Cet effet est différent de l'effet « classique » lié aux déformations uniformes se produisant sur une échelle macroscopique et qui donnent lieu à un déplacement de ces pics (contraintes résiduelles) [5].

Les microdéformations que l'on se propose de déterminer sont des nombres sans dimensions qui représentent l'écart quadratique moyen des déformations à l'échelle nanométrique, telles celles qui existent au voisinage des défauts ponctuels, des impuretés, des cœurs des dislocations, des surfaces, des joints de grains, etc. Elles traduisent l'écart du cristal réel par rapport au cristal parfait.

La méthode de Warren-Averbach [8] est plus précise pour séparer les deux effets : taille des domaines cohérents de diffraction et microdéformations. Malheureusement sa mise en œuvre est difficile car elle nécessite un traitement informatique sophistiqué. Une méthode approchée, dite de la «largeur intégrale », permet d'avoir accès aux ordres de grandeurs de ces deux effets. Elle donne les microdéformations avec une bonne précision, contrairement aux tailles des domaines cohérents de diffraction qui sont souvent imprécises. Dans cette étude, nous ne tiendrons compte que des premières.

1.1 MÉThode de La largeur intégrale. - Le but de cet article n'étant pas de présenter la partie théorique d'une telle méthode, nous renvoyons à la littérature $[6,9]$, et nous nous contenterons de préciser les grandes lignes de cette analyse.

On définit la largeur intégrale d'un pic de diffraction par :

$$
b(s)=\frac{\int I(s) \mathrm{d} s}{I_{\operatorname{Max}}(s)}
$$

où $I(s)$ est l'intensité diffractée pour le vecteur $s$ de l'espace réciproque, défini par

$$
s=\frac{2 \sin \theta}{\lambda}
$$

où $\theta$ est l'angle de diffraction et $\lambda$ la longueur d'onde des rayons $\mathrm{X}$.

L'élargissement des pics de diffraction est la superposition de trois effets qui peuvent être écrits :

$$
b(s)=\frac{1}{D}
$$

pour l'effet d'une taille des domaines cohérents de diffraction égale à $D$ dans la direction $s$ et

$$
b^{*}(s)=\sqrt{2 \pi} \varepsilon^{*} s
$$

pour l'effet de microdéformations de valeur $\varepsilon^{*}$

L'effet instrumental doit être déterminé expérimentalement. Une correction des largeurs intégrales est alors possible, mais nécessite une hypothèse sur le profil de la contribution instrumentale à l'élargissement. On peut émettre trois hypothèses différentes. Soit le pic est de 
type Cauchy $\left(I=\left(a+b \cdot s^{2}\right)^{-1}\right)$, soit de type Gauss $\left(I=b . \exp \left(-a^{2} s^{2}\right)\right)$, soit une combinaison de ces deux derniers. On nomme cette dernière correction, parabolique. Ces différents cas mènent à trois types de corrections :

$$
\begin{array}{ll}
\text { Cauchy : } & b=b_{\text {exp. }}-b_{\text {inst }} \\
\text { Gauss : } & b^{2}=b_{\text {exp }}^{2}-b_{\text {inst }}^{2} \\
\text { Parabolique : } & b=b_{\text {exp }}-\frac{b_{\text {inst. }}^{2}}{b_{\text {exp }}}
\end{array}
$$

où $b_{\exp }$ est la largeur expérimentale dans l'espace réciproque et $b_{\text {Inst }}$ la largeur instrumentale dans le même espace.

En ce qui concerne la séparation des effets de taille et de microdéformations, on refait les mêmes hypothèses concernant le type des profils. Ainsi, l'élargissement total du pic de diffraction, après correction instrumentale, est donné par la formule :

$$
b(s)=\frac{1}{D}+\sqrt{2 \pi} \varepsilon^{*} s
$$

si les deux effets sont de type Cauchy, et par la formule:

$$
b^{2}(s)=\frac{1}{D^{2}}+2 \pi \varepsilon^{* 2} s^{2}
$$

dans le cas où les deux effets sont de type Gauss.

Mais le cas le plus proche de la réalité utilise un profil de Cauchy pour l'effet de taille, et un profil de Gauss pour l'effet de microdéformations. Cela donne la formule empirique suivante (parabolique) :

$$
\frac{1}{b(s) D}=1-\frac{2 \pi \varepsilon^{* 2} s^{2}}{b^{2}(s)}
$$

1.2 MiSE en cEUVRE. - En général, l'analyse des microdéformations utilise deux pics de diffraction d'une même famille cristallographique (réalisés en géométrie $\theta-2 \theta$ ) mais pour deux ordres différents (par exemple, dans le cas de l'or, (111) et (222) voir Fig. 1). Ainsi, la valeur de $D$ représente la taille des domaines cohérents de diffraction dans la direction de croissance du film. Un logiciel permet de calculer la largeur intégrale des raies de diffraction. Deux raies analogues réalisées avec une poudre d'aluminium recuite donnent l'élargissement instrumental. En effet, si l'on suppose que cette dernière ne contient pas de microdéformations et que la taille des domaines est de plusieurs ordres de grandeur supérieure à celle mesurée dans les couches minces, l'élargissement observé est uniquement dû à l'effet instrumental.

On calcule alors les 9 valeurs correspondant aux différentes combinaisons des 3 hypothèses émises dans la correction et la séparation des effets. Le tableau I donne un exemple de calcul. Dans celui-ci, chaque ligne correspond à une hypothèse concernant la correction instrumentale et chaque colonne à une hypothèse concernant la séparation des effets. La valeur pour une correction et une séparation des effets de types paraboliques est la plus probable. L'écart, par rapport à cette valeur représente une majoration de l'erreur commise au niveau des hypothèses.

Bien que la méthode de la largeur intégrale puisse conduire à des valeurs absolues différentes de celles données par la méthode de Warren-Averbach, on peut toutefois l'utiliser pour étudier les variations relatives de ces valeurs en fonction de différents paramètres. L'ordre de grandeur des microdéformations ne semble pas varier significativement en fonction des hypothèses sur 


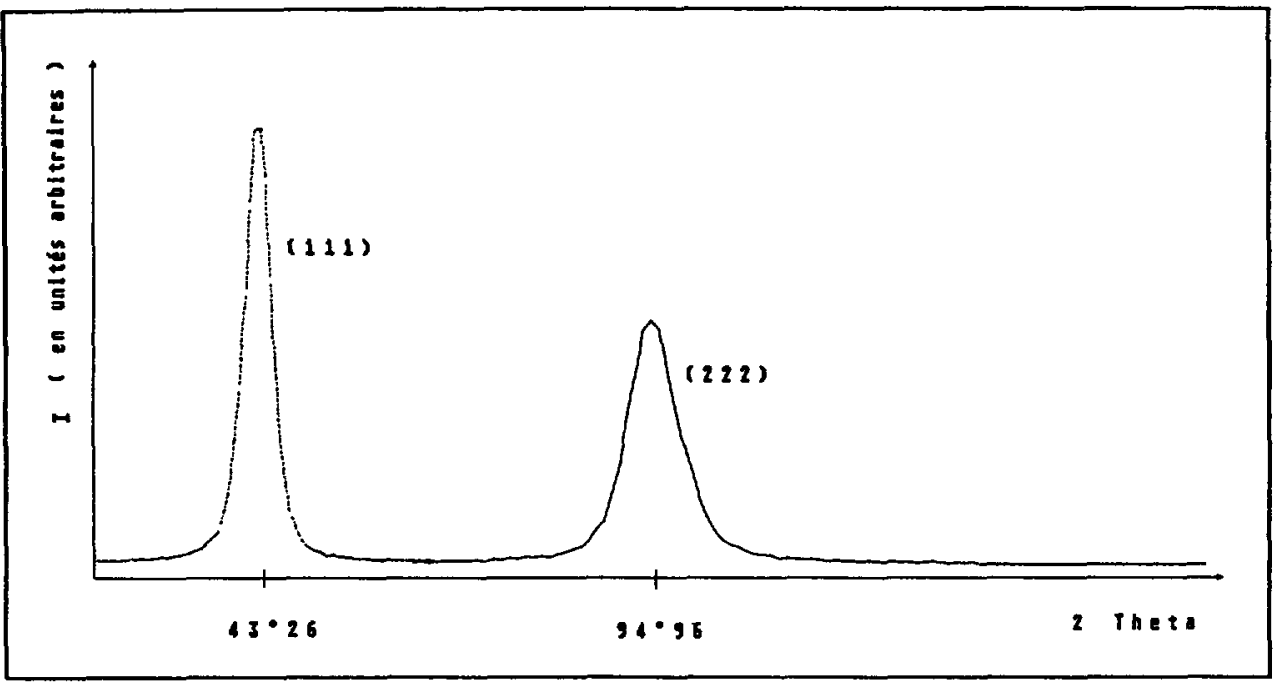

Fig. 1. - Pics de diffraction des plans (111) et (222) de l'Au pour l'échantillon 1.

[Au (111) and (222) planes diffraction peaks for sample 1.]

Tableau I. - Valeurs des microdéformations (a) et des tailles des domaines cohérents de diffraction (b) résultant des différentes combinaisons des hypothèses de la méthode de la largeur intégrale (Echantillon 2).

[Microdeformations (a) and diffracting coherent domains size (b) for the different hypothesis combinations of integral width method (sample 2).]

a) Microdéformations

\begin{tabular}{|l|l|l|l|}
\hline C.I. & S.E. Cauchy & S.E. Parab. & S.E. Gauss \\
\hline C.I. Cauchy & $2,56 \times 10^{-4}$ & $2,65 \times 10^{-4}$ & $2,97 \times 10^{-4}$ \\
\hline C.I. Parab. & $3,99 \times 10^{-4}$ & $4,23 \times 10^{-4}$ & $4,86 \times 10^{-4}$ \\
\hline C.I. Gauss & $4,22 \times 10^{-4}$ & $4,91 \times 10^{-4}$ & $5,99 \times 10^{-4}$ \\
\hline
\end{tabular}

b) Taille des Domaines (en $\mathrm{nm}$ )

\begin{tabular}{|l|c|c|c|}
\hline C.I. & S.E. Cauchy & S.E. Parab. & S.E. Gauss \\
\hline C.I. Cauchy & 700 & 449 & 372 \\
\hline C.I. Parab. & 326 & 220 & 193 \\
\hline C.I. Gauss & 147 & 113 & 107 \\
\hline
\end{tabular}


le profil du pic. En revanche, la valeur de taille des domaines $(D)$ est fortement dépendante de ces hypothèses. Il en résulte un intervalle d'erreur souvent du même ordre de grandeur que la valeur elle-même, c'est pour cela que nous ne considérerons pas les résultats de cette grandeur.

\section{Conditions expérimentales.}

2.1 PRÉPARATION DES ÉCHANTILlONS. - Nos échantillons sont réalisés par évaporation au canon à électrons, à température ambiante et sous un vide de $10^{-7}$ Torr. Nous avons utilisé deux types de substrats : du silicium monocristallin de $200 \mu \mathrm{m}$ d'épaisseur poli optique et de la silice fondue (plus rugueuse que le premier substrat).

Ce choix résulte d'une récente étude [10] qui a montré que la nature du substrat conditionne la texture des couches minces d'Au. Ainsi, conformément à cette étude, il est très difficile d'obtenir une couche de $150 \mathrm{~nm}$ texturée sur silicium, alors que la réussite sur silice fondue est de $100 \%$. Le tableau II donne les différents paramètres concernant les dépôts réalisés : vitesse de dépôt et épaisseur déposée.

Tableau II. - Conditions de dépôt des différents échantillons.

[Deposition conditions for the samples.]

\begin{tabular}{|c|c|c|c|}
\hline Echantillon & Substrat & Vitesse Dépôt & Epaisseur déposée \\
\hline 1 & Silicium & $1,5 \mathrm{~nm} / \mathrm{s}$ & $150 \mathrm{~nm}$ \\
\hline 2 & Silice Fondue & $0,1 \mathrm{~nm} / \mathrm{s}$ & $150 \mathrm{~nm}$ \\
\hline 3 & Silice Fondue & $0,5 \mathrm{~nm} / \mathrm{s}$ & $150 \mathrm{~nm}$ \\
\hline 4 & Silice Fondue & $1,5 \mathrm{~nm} / \mathrm{s}$ & $140 \mathrm{~nm}$ \\
\hline
\end{tabular}

2.2 UTILISATION DU RAYONNEMENT SYNCHROTON. - Toutes les mesures rapportées dans cet article ont été effectuées sur la ligne DW22 du LURE (Orsay, France). La brillance du rayonnement $X$, la qualité et la précision du diffractomètre quatre cercles de la ligne Wiggler d'une part, et la texture des couches minces étudiées d'autre part, ont contribué à faciliter l'analyse des microdéformations. La longueur d'onde utilisée pour cette étude est de $0,17387 \mathrm{~nm}$.

Parallèlement à cette analyse (tous nos échantillons étant polycristallins), nous avons effectué des déterminations de contraintes par diffraction des rayons $X$ selon la méthode des $\sin ^{2} \Psi$, méthode qui a déjà été largement présentée par ailleurs $[4,6]$. Cette dernière analyse nous permet également de déterminer le paramètre cristallin libre des contraintes $\left(a_{0}\right)$ qui nous renseigne directement sur la microstructure des couches, celui-ci étant influencé par les défauts ponctuels du cristal (impuretés, lacunes, interstitiels). Nous estimons les erreurs par la méthode statistique proposée par Kahloun [11]. L'ordre de grandeur de celles-ci est confirmé expérimentalement par répétition des mesures.

\section{Résultats et discussion.}

Le tableau III regroupe l'ensemble des résultats obtenus. La première remarque concerne les contraintes résiduelles : elles sont très faibles pour des couches minces brutes d'élaboration (de 
Tableau III. - Résultats obtenus par diffraction des rayons $X$. [X-Ray diffraction results.]

\begin{tabular}{|c|c|c|c|c|}
\hline Ech. & $(\mathrm{MPa})$ & $a_{0}(\mathrm{~nm})$ & Microdef. $\left(10^{4}\right)$ & Taille grains (nm) \\
\hline 1 & $27 \pm 15$ & $0,4087 \pm 0,0004$ & $10,6 \pm 1,19$ & $375 \pm 280$ \\
\hline 2 & $41 \pm 15$ & $0,4088 \pm 0,0004$ & $4,2 \pm 1,7$ & $220 \pm 113$ \\
\hline 3 & $-20 \pm 15$ & $0,4088 \pm 0,0004$ & $2,2 \pm 1,6$ & $231 \pm 112$ \\
\hline 4 & $-11 \pm 15$ & $0,4088 \pm 0,0004$ & $1,1 \pm 1,4$ & $235 \pm 111$ \\
\hline
\end{tabular}

-10 à $+40 \mathrm{MPa}$ ). On peut comparer ces résultats à ceux obtenus dans des films de tungstène de $100 \mathrm{~nm}$ d'épaisseur [4], ou dans des films de $200 \mathrm{~nm}$ d'acier inox [12], où il est usuel de trouver des contraintes de compression de l'ordre de plusieurs GPa. Nous pensons que la différence entre ces deux états de contraintes est liée à celle des énergies déposées par atome qui est de l'ordre de quelques électron-volts pour l'or et quelques dizaines d'électron-volts pour le tungstène et l'acier inox.

Du point de vue microstructural, nos échantillons se divisent en deux groupes:

- le premier concerne quatre films texturés obtenus principalement sur les substrats en silice fondue (groupe A);

- le second concerne 19 films non texturés, tous obtenus dans les conditions du tableau II sur un substrat de silicium recouvert d'une mince couche de silice (groupe B).

Le paramètre cristallin libre des contraintes $a_{0}$ a une valeur très différente dans les deux groupes. Il est de $0,4088 \pm 0,0001 \mathrm{~nm}$ pour le groupe $\mathrm{A}$, et $0,4067 \pm 0,0002 \mathrm{~nm}$ pour le groupe B. Le paramètre cristallin de l'Au massif est de $0,4074 \mathrm{~nm}$. En conséquence, les microstructures des deux groupes diffèrent non seulement par la texture, mais également par la nature des défauts ponctuels contenus dans la maille cristalline. Ils sont du type interstitiel dans le groupe $\mathrm{A}$ et lacunaire dans le groupe $\mathrm{B}$.

Il est également remarquable de constater que $a_{0}$ est très stable à l'intérieur de chaque groupe. Il ne semble pas dépendre des conditions opératoires du dépôt, contrairement au cas des films minces déposés par pulvérisation ionique [1] ou par voie chimique (C.V.D.) [13]. Ce point nous semble très important mais il n'y a, à notre connaissance, aucune étude sur $a_{0}$ dans le cas de films minces déposés au canon à électrons pour nous servir de référence ou de comparaison. Des études sur d'autres films déposés par le même procédé sont en cours actuellement.

Quant aux microdéformations déterminées pour le groupe A (voir Tabl. III), leur ordre de grandeur est comparable à celui des matériaux massifs. L'influence du substrat est ici clairement mise en évidence. Les microdéformations dans la couche déposée sur silicium sont très supérieures à celles observées dans le cas de la silice fondue. De même, l'augmentation de la vitesse de dépôt induit une forte décroissance des microdéformations montrant une évolution du réseau vers la perfection cristalline. Ainsi, l'écart quadratique par rapport à la structure moyenne (caractérisée par $a_{0}$ ) étant de plus en plus faible, la structure semble être de plus en plus uniformément centrée sur la valeur de $a_{0}$. Ces résultats expérimentaux démontrent donc un effet important de la cinétique du dépôt, sans qu'il soit pour l'heure, possible d'apporter de plus amples informations sur le phénomène. Toutefois, il est indéniable que les informations fournies par les microdéformations sont complémentaires de celles concernant le paramètre 
$a_{0}$. La détermination des microdéformations apporte donc des renseignements précieux sur l'état microstructural des films minces.

Ainsi, il ressort de tous ces résultats :

i) la nature du substrat et/ou la rugosité superficielle jouent un rôle fondamental dans l'apparition de la texture dans les films minces;

ii) le paramètre cristallin libre des contraintes, $a_{0}$, dépend fortement de la nature du substrat, mais contrairement au cas de films déposés par d'autres procédés, il ne dépend pas des conditions opératoires du dépôt par canon à électrons ;

iii) les microdéformations sont plus sensibles que le $a_{0}$ à la nature du substrat et aux conditions de dépôt. Elles donnent des informations complémentaires à celles obtenues par le $a_{0}$,

iv) la taille des domaines cohérents de diffraction déterminée par la méthode de la largeur intégrale est très sensible à l'hypothèse émise pour la déconvolution du pic de diffraction et ne semble pas fiable.

\section{Conclusion.}

En conclusion, nous avons démontré la faisabilité des mesures de microdéformations dans des films d'or texturés aussi minces que $150 \mathrm{~nm}$ et la sensibilité de cette grandeur aux paramètres de dépôt des couches étudiées. Bien que la méthode de la largeur intégrale utilisée ici ne soit qu'une méthode approchée, il est intéressant de noter que l'on a mis en évidence, grâce à elle, un effet des conditions d'élaboration sur l'état mécanique des dépôts, effet qui n'apparaît ni sur les contraintes résiduelles ni sur le paramètre cristallin sans contraintes, $a_{0}$. Ainsi, une nouvelle voie d'investigation, vers de nouvelles données concernant la microstructure de la phase diffractante et ainsi concernant les propriétés mécaniques des couches minces, est ouverte.

\section{Remerciements}

Les auteurs remercient MM. J. P. Lauriat et E. Elkaim (Responsables du poste expérimental WDIF4C) pour leur aide durant les expériences réalisées sur leur rayonnement synchrotron et M. Boisseau pour l'élaboration des échantillons.

\section{Bibliographie}

[1] Badawi K. F., Declemy A., Naudon A., Goudeau Ph.. Détermination des Contraintes Résiduelles par Diffraction des Rayons $X$ dans une couche mince de $1000 \AA$ de Tungstène, J. Phys. III France 2 (1992) 1741.

[2] Vink J. J., Somers M. A. J., Daams J. L. C. et al., Stresses, Strain and Microstructure of Sputterdeposited Mo Thin Films, J. Appl. Phys. 70 (1991) 4301.

[3] Leiberich A., Roy P. K., Crystallite Dimensions and Strain in Poly-Si and Ti-Silicide Thin Films Measured with Grazing Incidence X-Ray Diffraction, M.R.S. Proc. 239 (1991) 87.

[4] Durand N., Badawi K. F., Goudeau Ph., Naudon A., Evolution des Contraintes Résiduelles dans des Films Minces de Tungstène en fonction de l'Irradiation, J. Phys. III France 4 (1994) 25-34.

[5] Maeder G., X-Ray Diffraction and Stress Measurement, Chem Scripta 26A (1986) 23-31.

[6] Castex L., Lebrun J. L., Maeder G., Sprauel J. M., Détermination des Contraintes Résiduelles par Diffraction des Rayons X (Pub. Sci. Tech. ENSAM, Paris, 1981).

[7] Guinier A., Théorie et Technıque de la Radiocristallographie (Ed. Dunod, Paris, 1964).

[8] Warren B. E., Averbach B. L., The Effect of Cold-Work Distorsion on X-Ray Patterns, J. Appl. Phys. 21 (1950) 595. 
[9] Wagner C. N. J., Aqua E. N., Adv. X-Ray Analysis 7 (1964) 46.

[10] Golan Y., Marguli L., Rubinstein I., Pub. Surf. Scl., Weizman Institute of science (1991).

[11] Kahloun C., Badawi K. F., Diou A., Incertitude sur l'analyse des contraintes par diffraction des rayons X, Rev. Phys. Appl. 25 (1990) 1225.

[12] Boubeker B., Goudeau Ph., Eymery J. P., Badaw1 K. F., Détermination des Contraintes Résiduelles dans les Films Minces d'Acier Inoxydable 304L par diffraction des Rayons X, Soumis au $J$ Phys. III France.

[13] Ravet M. F., Badawi K. F., Durand N., Lafontaine H., Barnole V., Haghiri-Gosnet A. M., Intragranular microstructure and stress in sputtered tungsten thin films, Proc. M.R.S. (Spring Meeting, San Francisco, 1993). 